\section{Problematische Kakerlakensanierung}

\begin{abstract}
akerlakenallergene tragen in den USA zu einem nicht unerheblichen Teil zur Asthmaentwicklung bei Kindern bei. Großangelegte Sanierungsmaßnahmen mit dem Einsatz von Borsäure töteten zwar einen Großteil der Tiere, führten aber zu einem Anstieg der Allergene in den Wohnungen. Man vermutete, dass die nicht getöteten Tiere durch Kontakt mit subletalen BoräureDosen, z. B. durch Fressen toter Artgenossen, vermehrt Bla g 2 freisetzen.
\end{abstract}

Diese Vermutung bestätigte sich im Experiment an einer Kolonie mit $100 \mathrm{Ex}$ emplaren von Blattella germanica. Vermutlich greift Borsäure den Kiemendarm der Insekten an und führt so zu einer vermehrten Freisetzung der Protease Bla g 2 aus dem Verdauungssystem. Als Alternative zu Borsäure sollte daher auf Hydramethylnon zurückgegriffen werden.

$b k$

Zhang YC et al. Allergy 2005; 60: 965-8

\title{
Urtikaria durch Antiurtikaria-Mittel
}

En ntwickelt ein Urtikaria-Patient unter C Gabe eines $\mathrm{H}_{1}$-Blockers erneut die Symptome seiner Erkrankung, so deutet dies nicht unbedingt auf die Wirkungslosigkeit oder eine zu niedrige Dosis des Medikaments hin. Die Hauterscheinungen können auch eine Nebenwirkung des Antihistaminikums sein - ein seltenes, aber bekanntes Phänomen. Erstmals wurde es jetzt auch unter der Einnahme von Levocetirizin beschrieben. Eine 33jährige Urtikaria-Patientin hatte zunächst unter 10 bis $20 \mathrm{mg}$ Cetirizin generalisierten Pruritus und urtikarielle Eruptionen entwickelt, in der diagnos-

\section{Allergenarme Labradore}

1lergische Sensibilisierungen gegen
Hunde finden sich bei bis zu 8\%
der Bevölkerung. Die Allergenprodukti-
on dürfte von Hund zu Hund unter-
schiedlich sein, doch erst jetzt suchte ein
französisches Forscherteam systematisch
nach spezifischen Einflussfaktoren auf
die Allergenproduktion bei verschiede-
nen Hunderassen. Tatsächlich fanden sie
in den Fellproben der Testhunde stark
variierende Konzentrationen von Can f 1 .
Als relativ allergenarm erwiesen sich La-
bradore, während sich die Werte anderer
Rassen wie Pudel, Pinscher oder York-
shire-Terrier kaum unterschieden. Inner-
halb der Rassen tendierten jeweils die
männlichen Hunde zu einer höheren tischen Abklärung konnte die Symptomatik auch durch die zweimalige Gabe von $5 \mathrm{mg}$ Levocetirizin hervorgerufen werden. Dies sei wenig überraschend, kommentieren die Autoren, da es sich bei Levocetirizin um das L-Enantiomer des Razemats Cetirizin handelt. Beide Präparate haben den gleichen, als Antigen fungierenden Piperazinring. Das chemisch anders aufgebaute Loratadin wurde von der Patientin dagegen problemlos vertragen.

$b k$

Kränke B et al. Dermatology 2005; 210: 246-7

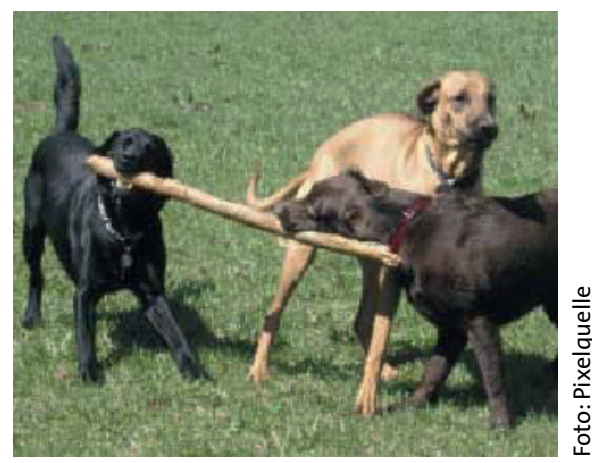

Allergenproduktion, die Haarlänge spielte keine Rolle. Signifikant korrelierte die Allergenproduktion dagegen mit einer Seborrhoe der Tiere.

$b k$

Ramadour M et al. Allergy 2005; 60: 1060-4

\section{Themenabend Allergie}

D er deutsch-französische Kultursender ARTE strahlt am Dienstag, den 28. März 2006, einen Themenabend Allergie aus. Beginnend um 20.45 Uhr werden drei Filme gezeigt: „Angriff der Allergene", eine Art Einführung und Überblick über die Volkskrankheit Allergie. Dann folgt ein Film über „Kinder mit Allergien", in dem besonders drastische Fälle porträtiert werden. Den Abend beschließt der Beitrag „Rätsel Allergie Spurensuche im Labor", der die aktuelle Allergieforschung beleuchtet.

www.arte.de 